Rogério G. dos Santos

rogerio.santos@em2c.ecp.fr

Laboratoire EM2C - CNRS / Ecole Centrale Paris,

France

\author{
José R. Figueiredo \\ jrigue@fem.unicamp.br \\ State University of Campinas - UNICAMP \\ Faculdade de Engenharia Mecânica \\ Cx.Postal 6122 \\ 13083-860 Campinas, SP. Brazil
}

\section{Laminar Elliptic Flow in the Entrance Region of Tubes}

The developing region of an axially symmetric laminar flow from a reservoir to a sharpedged tube is numerically simulated with a primitive-variables solver of the Navier-Stokes equations, using a plenum upstream of the tube inlet in order to avoid arbitrary specification of the profile at the inlet. Development region lengths, velocity profiles and head losses for varying Reynolds numbers are obtained. Results are compared to available experimental and theoretical results.

Keywords: laminar flow, developing flow, localized loss, tube inlet

\section{Introduction}

In hydraulic engineering, head losses in tubing are assumed to be formed by the sum of two parts, the "distributed" or "major" losses, and the "localized" or "minor" losses. The first part is associated to the well-known fully developed profiles, either laminar or turbulent, and is computed as if it covered the whole length of the tube. The second part is associated to departures from the developed profiles, such as those occurring in entrances, abrupt contractions or expansions, bends and other flow disturbances, and is usually dealt with by means of empirically determined coefficients for each of these geometries.

As widely recognized, the terms "major" and "minor" losses may be misleading, since in a short tube with several valves and bends the "minor" losses may well have a much larger combined effect then the "major" ones. Also, the term "localized" loss is somewhat artificial. In the entrance of a tube, for instance, the abrupt velocity profile at the beginning tends asymptotically to the developed profile within the so-called developing region, where the viscous dissipation is higher than that predicted by the developed profile. The "localized" loss is given by this increase in the viscous losses integrated through the developing region of the tube, together with small viscous losses upstream of the tube inlet.

Analogous situations hold for other disturbances of the developed profile. One consequence is that the accumulated effect of two or more sources of localized losses placed very close to each other is not the sum of the individual losses, as would be the case if they were sufficiently far apart for the developed flow to be reestablished before each localized loss.

Despite these well known facts, the distinction between distributed and localized losses is convenient for the practical computation of hydraulic circuits, being likely to remain the usual engineering practice.

Early theoretical approaches to the developing region used the boundary layer hypothesis of negligible diffusion along the streamline direction. The flow in the developing region is often interpreted as being formed by a core inviscid flow and a viscous layer adjacent to the walls which becomes thicker as the flow progresses until it reaches the center of the tube, where the core flow width vanishes. This qualitative description has also led to some of the earlier quantitative approaches to that problem. Another usual approach assumes the boundary layer hypothesis to hold in the entire developing region.

The practices based on the boundary layer theory lead to a parabolic equation that can be solved column by column in a

Paper accepted May, 2006. Technical Editor: Clovis R. Maliska. marching fashion, requiring the specification of the velocity profile at the tube inlet, where a uniform parallel flow is generally assumed. These approaches produced significant results with respect to the size of the developing region, but not with respect to the head losses, for the reasons given below.

The parabolic model does allow the computation of the pressure drop from the inlet to the fully developed zone. This can be complemented by assuming no dissipation upstream of the tube, so that Bernoulli's equation relates the pressures at the inlet and at an upstream stagnant point. However, as commented as early as 1968 by Astarita \& Greco, the unwarranted assumption about the inlet profile makes the calculation of the pressure drop through the tube unreliable, and the hypothesis of an ideal flow upstream of the tube inlet could be justified in a carefully designed, smooth entrance, but not in sharp corners.

More recent approaches towards this problem use either the creeping flow approximation for low Reynolds numbers or, more generally, the full elliptic Navier-Stokes equations, dismissing in both cases the boundary layer hypothesis. Although some of the solutions with the Navier-Stokes equations employ the same domain and the same entrance boundary conditions of the parabolic models, an important feature of applying the full Navier-Stokes equations to this problem is the possibility of using a plenum upstream of the tube inlet, so that the profile at the inlet can be determined, rather than arbitrarily postulated. Besides, the viscous losses upstream of the tube inlet can be considered.

The present study simulates the entrance region of the laminar stokesian flow emerging from a reservoir to a sharp-edged tube by adopting a large plenum whose entrance velocities are specified by two distinct radial flows towards the center of the tube inlet: an inviscid flow and a creeping flow. The comparison of these two solutions provides a direct indication of the influence of such choice, and of the reliability of the solutions obtained. The NavierStokes problem is solved numerically employing the CFD code PHOENICS, obtaining velocity profiles, development region lengths and head losses for Reynolds numbers between 1 and 2000. For each Reynolds number, solutions are achieved for several grid refinement levels, allowing the extrapolated value at infinite refinement to be estimated. The present results agree with the existing theoretical results for low Reynolds numbers and extend them for higher values. However, significant differences still persist with respect to the available experimental results; possible causes for such differences are raised, suggesting further investigations.

\footnotetext{
Nomenclature

$A=$ Area, $m^{2}$

$B=$ Auxiliary parameter, $\mathrm{m}^{5} / \mathrm{s}^{3}$
} 


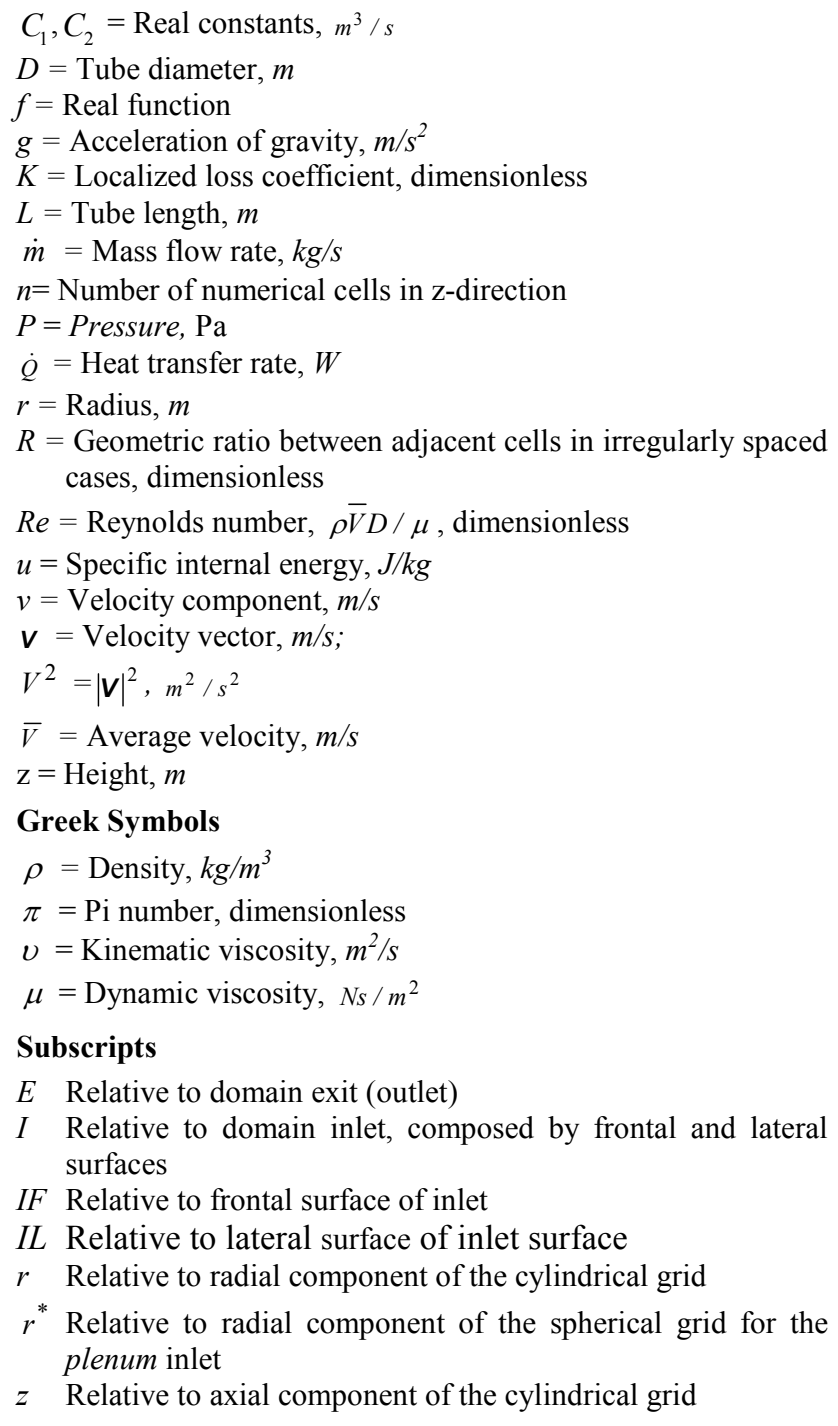

\section{Bibliographic Survey}

When dealing with localized head losses at tube inlets, most introductory textbooks on fluid mechanics present only an empirical coefficient valid for turbulent flows. Vennard \& Street (1978) is one of the few textbooks where the laminar case is referred to, stating only that the coefficient tends to decrease for crescent Reynolds numbers.

Experimentally determined head loss coefficients are presented by Idelchik (1969 e 1994). The 1969 edition shows a graphic of the localized head loss coefficient in sharp-edged tube inlets for Reynolds numbers between 10 and $10^{4}$; for lower Reynolds numbers it gives an algebraic expression, which is modified in the 1994 edition.

Astarita \& Greco (1968) performed an experimental study on head loss coefficient in sudden contractions of ratio 0.1616 $\left(D^{2} / D_{0}^{2}\right.$ ) for laminar flows, and found experimental results much higher than the available theoretical ones. Similar discrepancies were shown by Sylvester \& Rosen's (1970) results for contraction ratio 0.0156 and by Edwards, Jadallah \& Smith (1985), who determined the head loss coefficients for bends, valves, orifice plates and sudden contractions and expansions with contraction ratios 0.445 and 0.660 .

Perry, Green \& Maloney (1997) express the head losses in terms of equivalent tube lengths as a function of the Reynolds number for laminar flows through sudden contractions with aspect ratios smaller than 0.2 .

Schlichting (1979, Chapters IX and XI) reviews the theoretical studies based on the boundary layer hypothesis dealing with flows in the entrance regions of channels and tubes, including complex whirly flows.

The creeping flow approximation for very low Reynolds numbers has been considered by Benson \& Trogdon (1985) and by Sisavath et al (2002). Benson \& Trogdon used an analytic eigenfunction method to obtain the developing flow in a tube assuming two distinct profiles at the inlet: the uniform profile with slipping at the wall and the linear profile adherent to the wall with a maximum at the center. Sisavath et al considered the head losses in sudden expansions and contractions, which coincide in the case of creeping flow since their respective flows are symmetric to each other; their analytical results were in good agreement with the numerical results obtained by Oliveira, Pinho \& Schulte (1998), Vrentas \& Duda (1973) and by themselves.

Full Navier-Stokes equations and plenums upstream of the tube inlet were used by Vrentas \& Duda (1973), Sparrow \& Anderson (1977), Naylor et al. (1991), Sadri \& Floryan (2002a, 2002b), and Uribe (2002).

Vrentas \& Duda (1973) analyzed the sudden contraction of a tube by solving the Navier-Stokes equations under the vorticitystream function approach using an explicit finite difference scheme, obtaining head losses, development lengths and velocity profiles for Reynolds numbers $0,50,100$ and 200 and contraction aspect ratios 1.5, 2.5 and 4.0.

Sparrow \& Anderson (1977) also employed the vorticity-stream function approach to solve numerically the flow from a great reservoir to a duct for Reynolds numbers 1, 10, 50, 100, 300 and 1000 , and found the influence of the upstream flow upon the profile at the inlet to be more significant as the Reynolds number decreases.

The works on the entrance region of plane channels performed by Naylor et al. (1991) and by Sadri \& Floryan (2002a, 2002b) employ plenums with entrance boundary conditions defined by the Jeffery-Hamel flow. Their numerical grids are based on polar coordinates in the plenum and cartesian coordinates in the channel. Naylor et al. studied the natural convection between isothermal vertical plates by solving the Navier-Stokes and energy equations with the finite element program FIDAP. Sadri \& Florian studied the entrance flow in a channel using finite differences and the vorticitystream function approach, detecting a contraction vane for Reynolds numbers above 137.

The same problem was considered by Uribe (2002), also using a plenum with Jeffery-Hamel flow as entrance condition. However, sharp variations in the numerical grid were avoided by using Cartesian coordinates both in the plenum and in the channel. A finite-volume primitive variables approach was adopted, explicit for the velocities and implicit for the pressure. Central differencing was used when it was stable. For Reynolds numbers 100 to 400 both the exponential and the QUICK discretization schemes were employed, and the results from QUICK were considered more reliable. A velocity peak was found close to the walls in the beginning of the tube; a recirculation region was detected just downstream of the inlet for all Reynolds numbers studied.

The head loss coefficient in an axially symmetric sudden expansion was numerically computed by Oliveira, Pinho \& Schulte (1998) by means of a finite volume method over a co-located nonorthogonal grid with the upwind scheme for the convective terms and central differencing for the diffusive ones, obtaining results for Reynolds numbers between 0.5 and 200 and aspect ratios between 1.5 and 4.0 . 


\section{Physical Problem}

The computational domain is sketched in Fig.1, showing the physical walls of the tube and the reservoir (full and broken lines) as well as the fictitious boundaries at the inlet and outlet (dotted lines). Assuming the flow to be axisymmetric, the cylindrical reference grid $(r, z)$ is the most appropriate for the numerical computations on the tube. For geometric simplicity in this reference grid, the plenum upstream of the tube was established as a cylinder coaxial with the tube. The spherical reference grid $\left(r^{*}, \theta\right)$ is useful for analytically computing the inlet conditions prescribed at the entrance of the plenum.

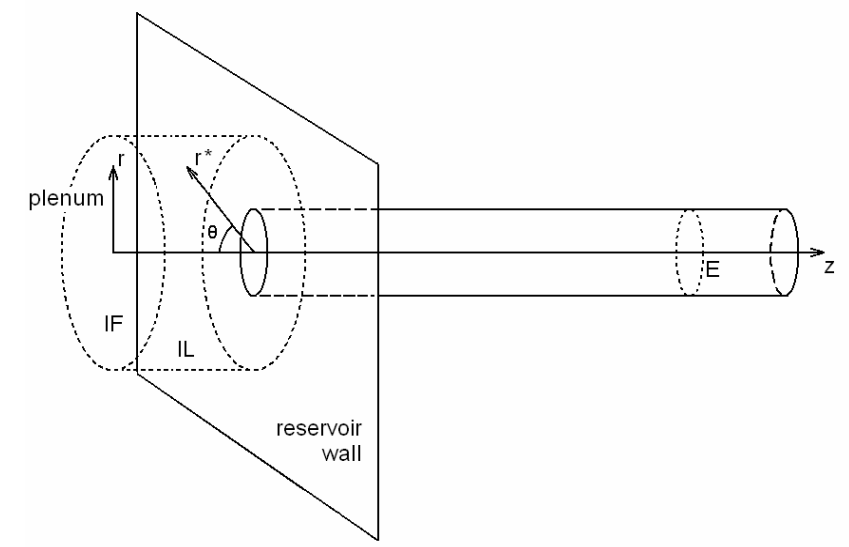

Figure 1. Sketch of computational domain, defined by frontal (IF) and lateral (IL) surfaces of the plenum, part of the reservoir wall, tube wall and exit surface (E).

Steady-state incompressible flow with null tangential velocity was assumed. Constant viscosity and isothermal conditions are also considered, neglecting the mild effects of the dissipation upon the thermal state. The governing equations are the continuity and the Navier-Stokes equations in the following conservative form:

$$
\begin{aligned}
& \frac{1}{r} \frac{\partial}{\partial r}\left(r v_{r}\right)+\frac{\partial v_{z}}{\partial z}=0 \\
& \rho\left(\frac{1}{r} \frac{\partial\left(r v_{r}^{2}\right)}{\partial r}+\frac{\partial\left(v_{z} v_{r}\right)}{\partial z}\right)=-\frac{\partial P}{\partial r}+ \\
& +\mu\left[\frac{\partial}{\partial r}\left(\frac{1}{r} \frac{\partial}{\partial r}\left(r v_{r}\right)\right)+\frac{\partial^{2} v_{r}}{\partial z^{2}}\right]+\rho g_{r} \\
& \rho\left(\frac{1}{r} \frac{\partial\left(r v_{r} v_{z}\right)}{\partial r}+\frac{\partial\left(v_{z}^{2}\right)}{\partial z}\right)=-\frac{\partial P}{\partial z}+ \\
& +\mu\left[\frac{1}{r} \frac{\partial}{\partial r}\left(r \frac{\partial v_{z}}{\partial r}\right)+\frac{\partial^{2} v_{z}}{\partial z^{2}}\right]+\rho g_{z}
\end{aligned}
$$

The positions of the boundaries at the inlet and outlet of the domain are somewhat arbitrary. For the exit boundaries, the numerical tube size was fixed as twice the development size established by Shah \& London (1978) for each Reynolds number considered. The numerical solution obtained confirmed the sufficiency of this choice of length.

Domain inlet conditions are generally more influential on the flow than the outlet conditions, particularly for convectiondominated flows, and there is no previous estimation of the required length of the plenum upstream the tube inlet. Arbitrarily setting the plenum length equal to its radius, a series of preliminary tests indicated that the profile at the tube entrance turned almost independent on the plenum size if the plenum radius was at least five times the tube radius; these proportions were adopted for all cases.

This question is linked to the profile imposed at the plenum entrance. In the present approach, the domain entrance and exit boundaries are specified in terms of velocities. Null derivatives were specified at the exit. The inlet conditions are of specified value, and two approaches were comparatively employed, each associated to a distinct model of the flow approaching the tube inlet, interpreted as a sink for the plenum flow. Both models assumed spherically radial flows towards the center of the tube inlet, so that the radial velocity is set in any case as $v_{r^{*}}=f(\theta) / r^{* 2}$. One model considers the uniform radial flow, which is a classic inviscid solution defined by $f(\theta)=C_{1}$, slipping on the reservoir walls. The other model emerges from a creeping flow solution that gives $f(\theta)=C_{2}(1+\cos 2 \theta)$, obeying the non-slip condition at these walls. The constants $C_{1}$ and $C_{2}$ are fixed in terms of the desired Reynolds number, as shown in detail by Santos (2004). The velocities to be imposed at the plenum entrance are computed on the spherical reference coordinates and subsequently projected onto the plenum cylindrical coordinates. As will be seen, both models produced practically the same profile at the tube inlet, with minor differences with respect to head loss.

For computing the head loss due to the sharp entrance, one applies the first law of Thermodynamics to the control volume defined by the numerical domain, obtaining:

$$
\begin{aligned}
& -\int_{I}\left(\frac{V^{2}}{2}+g z+\frac{P}{\rho}\right) \rho \boldsymbol{V} d \boldsymbol{A}-\int_{E}\left(\frac{V^{2}}{2}+g z+\frac{P}{\rho}\right) \rho \boldsymbol{V} d \boldsymbol{A}= \\
& =-\dot{Q}+\int_{I} \rho u \boldsymbol{V} d \boldsymbol{A}+\int_{E} \rho u \boldsymbol{V} d \boldsymbol{A}
\end{aligned}
$$

As usual, the vector elementary area $d \boldsymbol{A}$ is directed outwards the domain. The left member of the above equation shows the head loss as the difference between the sums of kinetic, potential and pressure energies at the inlet and at the outlet of the domain, expressing the destruction of these thermodynamically noble forms of energy. The right member represents the same head loss as the thermal energy it turns into, i.e., either as increased internal energy or as heat transferred to the surroundings. These thermal forms of energy are unimportant for the incompressible case. Instead, the head loss is computed as the sum of distributed and localized losses, as can be found in any standard textbook in fluid mechanics (see, for instance, Fox \& McDonald, 1988), yielding:

$$
\begin{aligned}
& -\int_{I}\left(\frac{V^{2}}{2}+g z+\frac{P}{\rho}\right) \rho \boldsymbol{V} d \boldsymbol{A}-\int_{E}\left(\frac{V^{2}}{2}+g z+\frac{P}{\rho}\right) \rho \boldsymbol{V} d \boldsymbol{A}= \\
& =\dot{m}\left(\frac{64}{\operatorname{Re}} \frac{L}{D} \frac{\bar{V}_{E}^{2}}{2}+K \frac{\bar{V}_{E}^{2}}{2}\right)
\end{aligned}
$$

The first term within the parenthesis on the right side of Eq. (5) corresponds to the distributed loss for the laminar case, and the second term to the localized loss at the tube inlet. Solving Eq. (5) for the localized loss coefficient $K$ one obtains:

$$
K=\frac{4 \pi \rho}{\dot{m} \bar{V}_{E}^{2}} B-\frac{64 L}{D^{2} \bar{V}_{E} U^{-1}}
$$

where $B$ is an auxiliary parameter given by: 


$$
\begin{aligned}
& B=r_{I L} \int_{I L}\left(\frac{V^{2}}{2}+g z+\frac{P}{\rho}\right) v_{r} d z+\int_{I F}\left(\frac{V^{2}}{2}+g z+\frac{P}{\rho}\right) v_{z} r d r \\
& -\int_{E}\left(\frac{V^{2}}{2}+g z+\frac{P}{\rho}\right) v_{z} r d r
\end{aligned}
$$

The squared velocity module $V^{2}=|\vec{V}|^{2}$ is computed as the sum of the squared velocity components $\left(v_{r}^{2}+v_{z}^{2}\right)$. The above integrations are performed numerically after the converged solution is found, for various grid refinement levels. The final coefficient is obtained by means of the Richardson's extrapolation for vanishing grid sizes.

\section{Numerical Solution}

The numerical problem was solved employing the proprietary CFD code PHOENICS, which operates with primitive variables using the SIMPLEST procedure to relate the pressure and the continuity equation.

The numerical grid was regular in the $r$-coordinate on the whole domain and also regular in the $z$-coordinates within the plenum. For lower Reynolds numbers, where the developed profile is achieved in short distances, the grid was also regular in the tube, with the same spacing used in the plenum. Only for Reynolds numbers of 20 and above 20, that required large tube sizes to achieve the developed profile, was it found advantageous to employ irregular grids in the $z$ coordinate within the tube, so decreasing the number of cells. In these cases, the first cell in the tube had the same size of the cells in the plenum and the remaining cell sizes in the tube were set geometrically crescent.

The code also offers another grid size variation rule, called power rule, which was avoided because it leads to cells sizes varying, in relative terms, very strongly in the beginning of the tube and very smoothly afterwards, while the geometric rule imposes a uniform rate of variation across the space.

In order to keep the same convergence characteristics of the regularly spaced case, the refinement studies used the cell size geometric ratio $R$ decreasing with the number of $z$-direction cells $n$ in such a way that the product $n \ln R$ was kept constant. This criterion was inspired by Rivas' (1972) demonstration that the quadratic spatial convergence of the central differencing scheme is maintained for irregular grids, provided that during the refinement process there is a unique function with bounded non-null derivatives mapping a regularly spaced transformed domain into the irregularly spaced physical domain. The above referred criterion was shown to hold for many other quadratic discretization schemes by a simple numerical test due to Figueiredo and Llagostera (2000, also Llagostera and Figueiredo, 2000).

The last decades saw extensive research on numerical discretization schemes for the momentum equations and other convective-diffusive transport equations. So far, central differencing remains a sufficiently accurate scheme if it is stable, and, for high Reynolds numbers, the QUICK scheme emerges as one of the most successful. This conclusion was confirmed for entrance flows in channels by Uribe (2002).

The UNIFAES scheme (Figueiredo, 1997) has shown generally superior accuracy, and always superior stability and robustness in comparison with both central differencing and QUICK (Vilela, 2001). However, while central differencing and QUICK are incorporated, together with other schemes, to the PHOENICS code, the new scheme UNIFAES has not been implemented in any commercial code so far.
Accordingly, the present computations employed the central differencing scheme for Reynolds numbers up to 100. The QUICK scheme for Reynolds numbers of 100 and above, where the central differencing did not converge. Very close results were obtained with the two schemes for the common case of Reynolds number equal to 100 .

The convergence criteria in each case was such that the result is not affected by lower levels of tolerance.

\section{Results}

Fig. 2 shows the axial and radial velocity component profiles obtained at the tube entrance assuming both inviscid and creeping flows at the plenum inlet for Reynolds number equal to 100 . The two profiles are visually identical for both boundary conditions, in a clear indication that the plenum size is sufficient.

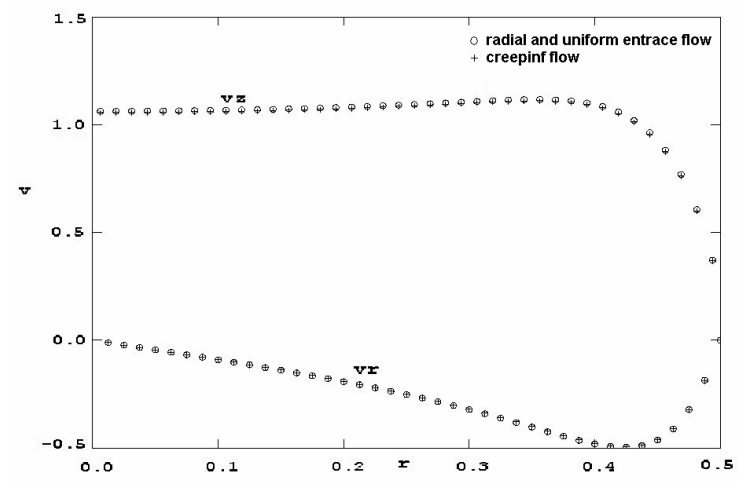

Figure 2. Axial and radial velocity components on east plenum entrance for $\mathrm{Re}=100$.

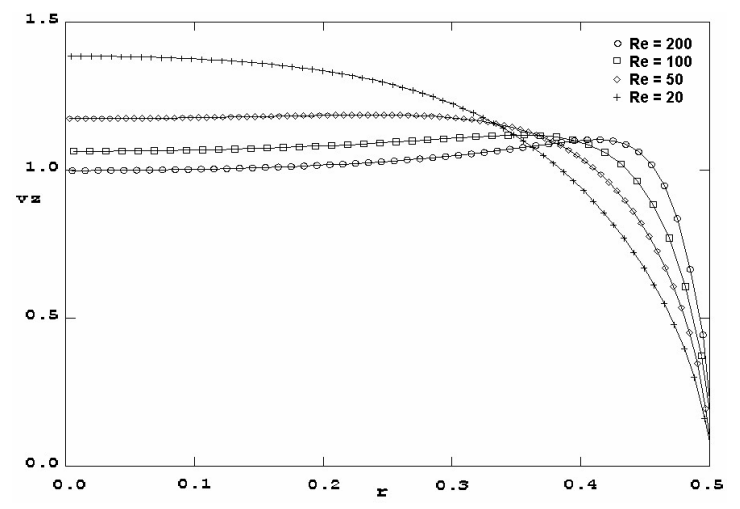

Figure 3. Axial velocity profiles at tube inlet for Re between 20 and 200.

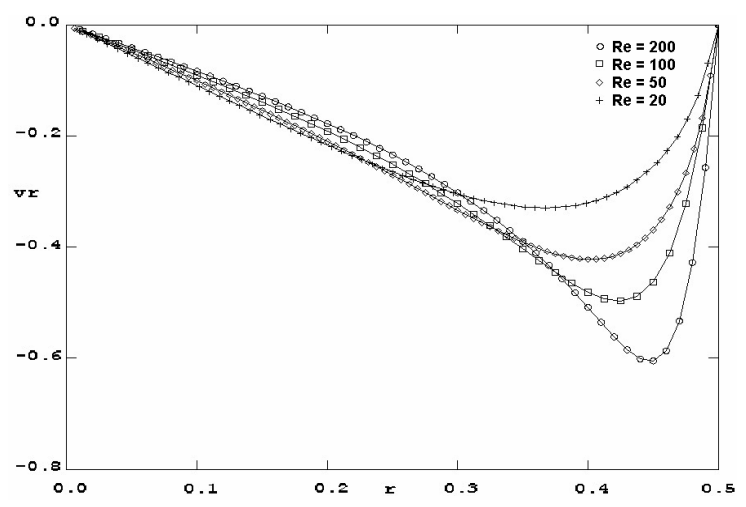

Figure 4. Radial velocity profiles at tube inlet for Re between 20 and 200. 


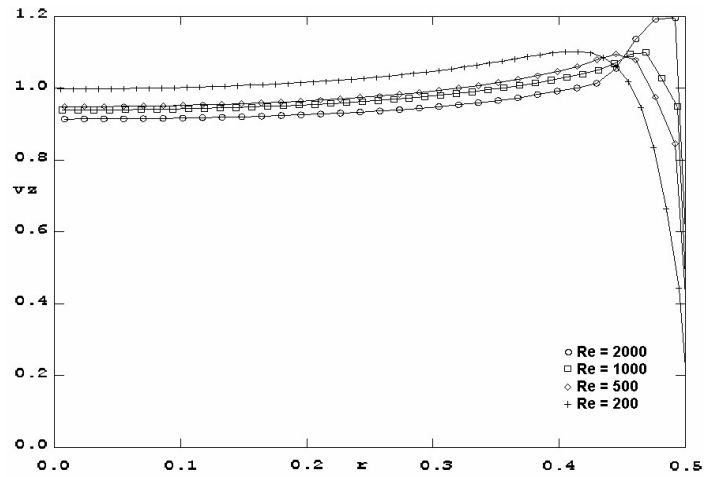

Figure 5. Axial velocity profiles at tube inlet for Re between 200 and 2000.

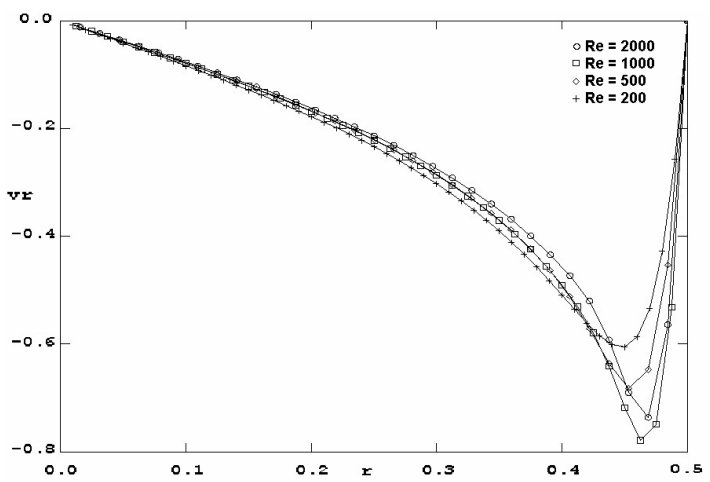

Figure 6. Radial velocity profiles at tube inlet for $\mathrm{Re}$ between 200 and 2000.

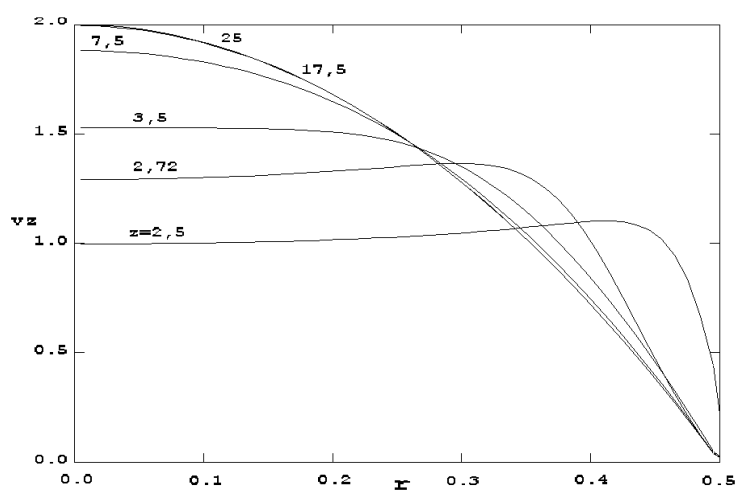

Figure 7. Developing axial velocity profiles for $\mathbf{R e}=\mathbf{2 0 0}$.

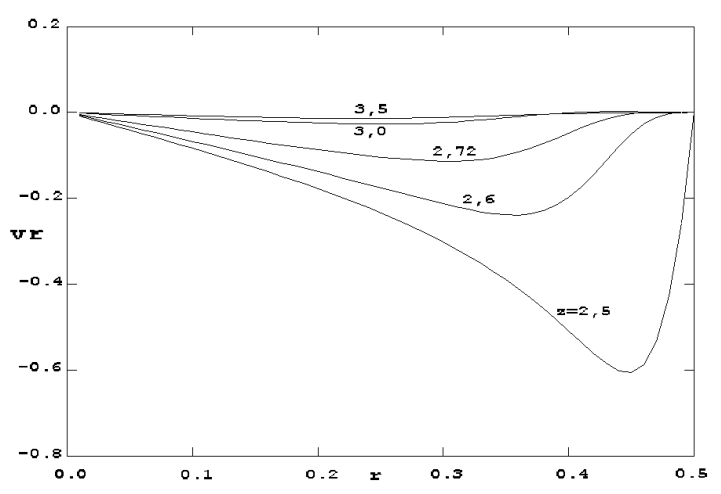

Figure 8. Developing radial velocity profiles for $\mathrm{Re}=\mathbf{2 0 0}$.

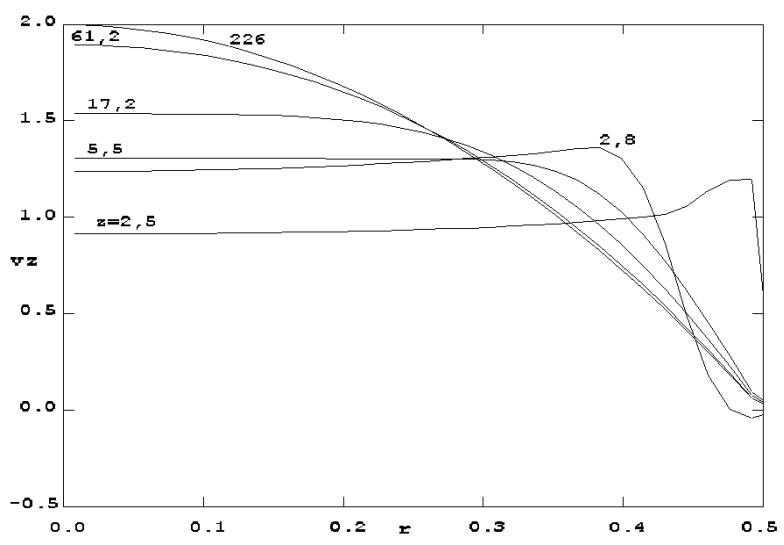

Figure 9. Developing axial velocity profiles for $\mathbf{R e}=\mathbf{2 0 0 0}$.

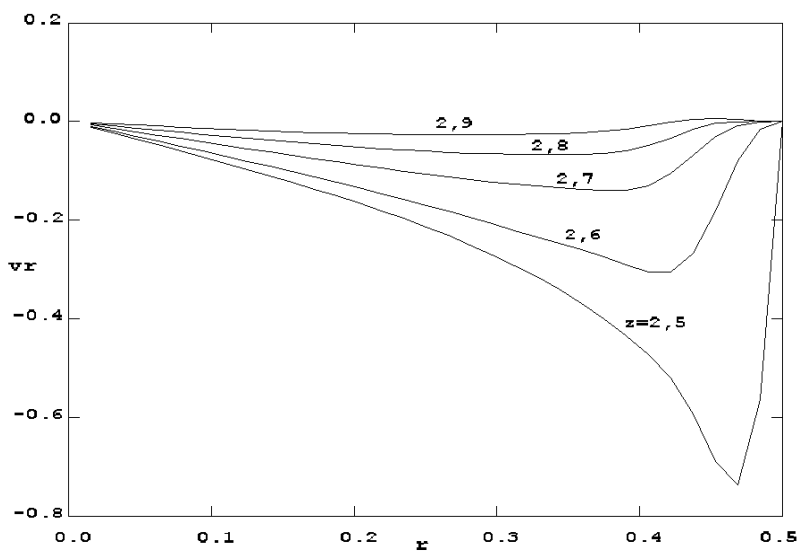

Figure 10. Developing radial velocity profiles for $\mathbf{R e}=\mathbf{2 0 0 0}$.

Figures 3 to 6 show the evaluation of the inlet profile for variable Reynolds numbers. For Reynolds numbers greater than 100 , the axial velocity component develops a peak that becomes more pronounced and closer to the tube wall as the Reynolds number increases. The radial velocity component also develops a negative peak in about same place, and its magnitude reaches about half the magnitude of the axial velocity, showing that it is by no means negligible as the parallel flow assumes.

The evolution of the velocity profiles along the tube is shown in Fig. 7 to 10 for Reynolds numbers 200 and 2000. For $R e=200$ the initial peak velocity has entirely disappeared at the distance of $1 \mathrm{D}$ from the entrance, while for $\mathrm{Re}=2000$ a distance equivalent to $3 \mathrm{D}$ was necessary. For Reynolds number 2000, as well as for 1000, a recirculating zone is detected in the entrance.

The length of the developing region, defined as the distance from the tube inlet to the point where the centerline velocity achieves $99 \%$ of its developed value, is plotted in Fig. 11 for varying Reynolds numbers, together with the developing lengths obtained by Shah \& London (1978) and by Dombrowki et al. (1993) using the parabolic model. Clearly, all results are very similar for moderate and high Reynolds numbers, and differ sensibly for low Reynolds numbers, representing creeping and other flow situations where the parabolic model is not applicable. 


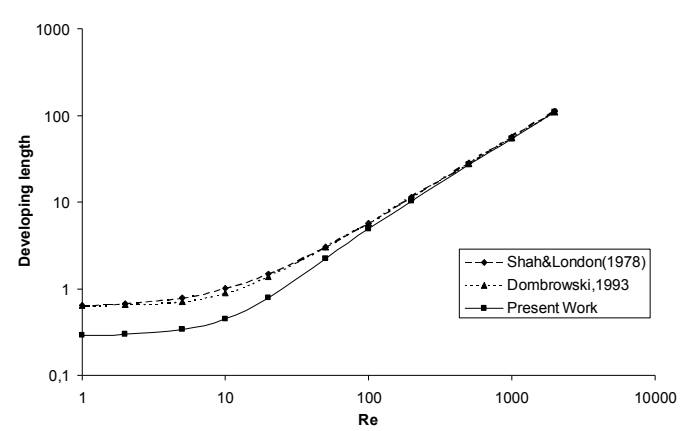

Figure 11. Developing length.

The values of the development region length found here can also be expressed algebraically in a form analogous to that presented by Dombrowski et al. as follows:

$$
L / D=0.8476 \exp (-0.05869 \mathrm{Re})+0.05456 \mathrm{Re}-0.5640
$$

The determination of the localized loss coefficient requires very precise computations, since it results from the difference between the integrated noble forms of energy in the entrance and the exit of the domain, also subtracting the loss due to the developed profile. Indeed, this parameter turned out to be very sensible to grid refinement, as shown in figures 12 and 13 for Reynolds numbers 200 and 2000 respectively. Five different levels of refinement are reported for each Reynolds number and each assumed profile at the entrance of the plenum. The most refined computation in the case $\mathrm{Re}=200$ employed 650 nodes in $z$-direction and 250 nodes in $r$ direction in the plenum, and in the case $\mathrm{Re}=2000$ it employed 544 and 160 nodes respectively.

Despite the very refined mesh employed, a grid dependent result still appears at the entrance of the tube for Reynolds number 2000 (Figures 5 and 6 , and $\mathrm{z}=2.5$ in Figures 9 and 10). This numerical error disappears entirely within the short distance of $0.1 \mathrm{D}$ from the entrance $(z=2.6)$.

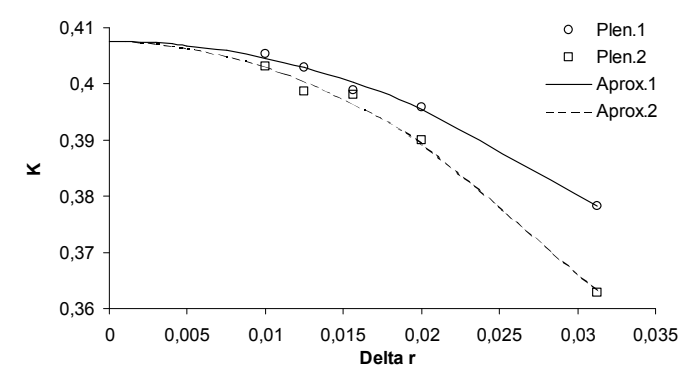

Figure 12. Localized loss coefficient as function of grid refinement for $\mathrm{Re}=\mathbf{2 0 0}$.

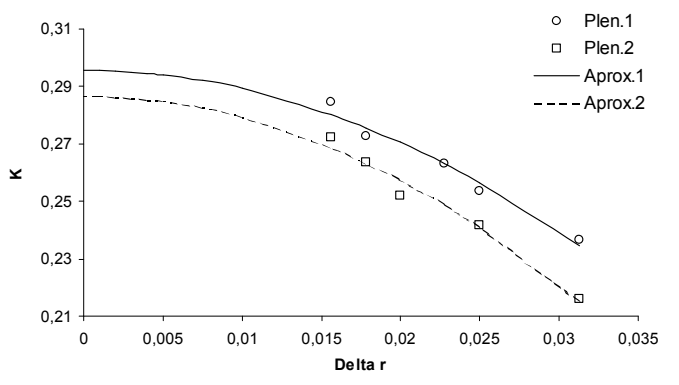

Figure 13. Localized loss coefficient as function of grid refinement for $\operatorname{Re}=2000$.

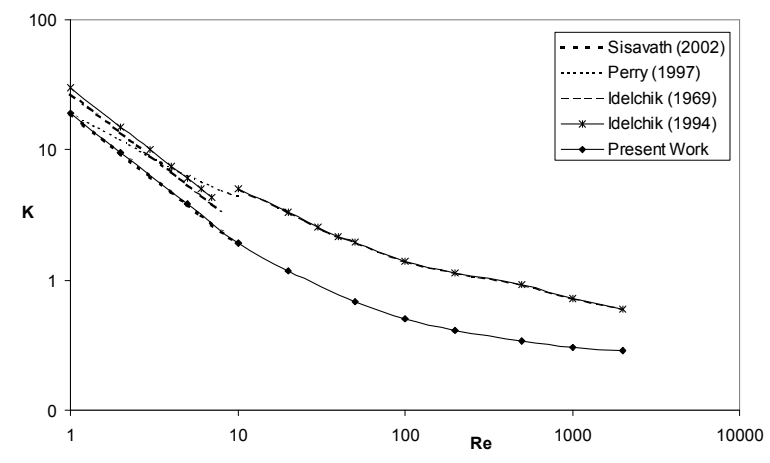

Figure 14. Localized loss coefficient.

The lines shown in Fig. 12 and 13 are obtained by fitting a curve of type $K=K_{0}+\alpha \cdot \Delta x^{2}$, according to the Richardson's extrapolation; quadratic spatial convergence was assumed as suggested by the second-order schemes employed (see for instance Morton \& Mayers, 1996, section 6.8) and also because curves assuming linear convergence did not fit the calculated points so closely. However, the quadratic fitting is not exact, possibly indicating random errors such as round-off, etc.

Curiously, the extrapolated value at infinite refinement, $K_{0}$, obtained using the creeping flow (Plen.1) and the inviscid flow (Plen.2) profiles at the plenum entrance, are practically coincident for most Reynolds numbers, as exemplified for the case $\mathrm{Re}=200$. The case $\mathrm{Re}=2000$ is the most important exception, yet the two results are not far apart $\left(K_{0}=0.287\right.$ and 0.295$)$. This is further evidence that the plenum size is sufficient. For all Reynolds numbers considered and all finite refinements, the solution obtained with the creeping flow profile was higher and closer to the estimated converged solution at infinite refinement.

Figure 14 plots the localized loss coefficient so obtained as a function of the Reynolds number, together with the theoretical results presented by Perry et al. (1997), Sisavath et al. (2002), and an extrapolation of the experimental results on sudden contractions presented by Idelchik (1969 and 1994) to the limiting case of infinite ratio. The present results closely agree with the results analytically obtained by Sisavath et al. under the creeping flow hypothesis; however, results presented by Perry et al. are not so close. The experimental results due to Idelchik bear some resemblance to, and significant differences from, the theoretical results obtained here and elsewhere: the experimental head loss coefficient also decays with the Reynolds number, but its value is between twice and three times the theoretical one, and it exhibits a discontinuous increase for a Reynolds numbers of about 10 .

The present authors have no elements to question the experimental results. Instead, it is necessary to raise the possibility that the hypothesis employed in the present computations of a steady-state axysimmetric flow should be questioned. Therefore, two possible reasons for the differences between all theoretical results and the experimental ones appear: 1- the presence of oscillations in the flow, increasing the mean head loss, 2- the occurrence of non symmetric results, occasionally more stable than the assumed symmetric results.

\section{Conclusions}

The problem of the laminar flow in a sharp edged tube emerging from a large reservoir was numerically simulated by solving the full elliptic Navier-Stokes equations assuming axially symmetric flow, and using a plenum upstream of the inlet so that the velocity profile at the tube inlet could be calculated instead of postulated. 
Velocity profiles obtained at the tube inlet were very distinct from the parallel profile often assumed, exhibiting a velocity peak close to the walls for Reynolds numbers equal or above 200 and, perhaps more importantly, showing a radial component whose maximum value reaches about half the axial velocity values. Furthermore, results indicated possible recirculations for the highest Reynolds numbers studied.

The developing region length determined here closely agrees with that presented by Shah \& London (1978) and by Dombrowki et al. (1993) for moderate and high Reynolds numbers, and differs only for low Reynolds numbers (creeping flow situations).

The localized head losses obtained here are very close to those obtained theoretically by Sisavath et al. (2002) for creeping flows, but sensibly distinct from the experimental values obtained by Idelchik $(1969,1994)$. The curve of the localized head loss at the entrance against the Reynolds numbers bears some resemblance to the experimental curve, but quantitatively it is about two or three times lower than the experimental results.

As detailed throughout the text, much care was exercised in computing the head loss at the duct entrance: the use of a large plenum upstream of the flow, the comparative use of two different inlet conditions for such plenum, the large tube length, the choice of the best discretization scheme available in the package employed, the high level of grid refinement, the continuity of the grid size about the entrance and everywhere, the attention to Rivas's (1972) conditions for quadratic convergence of irregular grid, and the use of Richardson's extrapolation. In view of these precautions, it was concluded that no flaw in the two-dimensional computation model can account for the difference between theoretical and experimental results. Therefore, further research is demanded to investigate the difference between all theoretical results and experimental data. The two hypothesis raised to explain this phenomena - the presence of oscillations in the flow, and the occurrence of non symmetric solutions - can only be numerically investigated by using threedimensional flow models, and the oscillations also require transient codes.

\section{References}

Astarita, G. \& Greco, G., 1968, "Excess Pressure Drop in Laminar Flow Through Sudden Contraction", Ind. Eng. Chem. Fundam, Vol. 7, No. 1, pp. 27-31.

Benson, L.H. \& Trogdon, S.A., 1985, "An eigenfunction solution of entry flow in a semi-infinite pipe at low Reynolds numbers", Applied Scientific Research, Vol. 42, pp. 347-359.

Dombrowski, N., Foumeny, E.A., Ookawara, S. and Riza, A., 1993, "The Influence of Reynolds Number on the Entry Length and Pressure Drop for Laminar Pipe Flow", The Can. J. of Chemical Engineering, Vol. 71, pp. 472-476.

Edwards, M.F., Jadallah, M.S.M. and Smith, R., 1985, "Head Losses in Pipe Fittings at Low Reynolds Numbers", Chem. Eng. Res. Des., Vol. 63, pp. 43-50.

Figueiredo, J.R. \& Llagostera, J., 2000, "Numerical Study on Mixed Convection in a Horizontal Flow Past a Square Porous Cavity using
UNIFAES Scheme”, J. Brazilian Soc. Mechanical Sciences, Vol. XXII, No. 4, pp. 583-597.

Figueiredo, J.R., 1997, “A Unified Finite-Volume Finite-Differencing Exponential-type Scheme For Convective-Diffusive Fluid Transport Equations", J. Brazilian Soc. Mechanical Sciences, Vol. XIX, No. 3, pp. 371-391.

Fox. R. W. \& McDonald, A. T., 1988, Introduction to Fluid Mechanics (in Portuguese), Guanabara Koogan, Rio de Janeiro.

Idelchick, I.E., 1969, Memento des Pertes de Charge Eyrolles, Paris.

Idelchick, I.E., 1994, Handbook of Hydraulic Resistance, CRC Press, Boca Raton, $3^{\text {rd }}$ ed.

Llagostera, J \& Figueiredo, J.R., 2000, “Application of the UNIFAES Discretization Scheme to Mixed Convection in a Porous Layer with a Cavity, Using the Darcy Model", J. Porous Media, Vol. 3, No. 2, pp. 139154.

Morton, K.W. \& Mayers, D. F., 1996, Numerical Solution of Partial Differential Equations, Cambrige University Press, Cambridge

Naylor, D., Floryan, J.M. and Tarasuk, J.D., 1991, “A numerical Study of developing free convection between isothermal vertical plates", Journal of Heat Transfer, Vol. 113, pp. 620-626.

Oliveira, P.J., Pinho, F.T and Schulte, A., 1998, “A General Correlation for the Local Loss Coefficient in Newtoniam Axisymmetric Sudden Expansions", International Journal of Heat and Fluid Flow, Vol. 19, pp. 655-660

Perry, R.H., Green, D.W. and Maloney, J.O., 1997, Perry's Chemical Engineers'Handbook, McGraw-Hill, New York, 7th ed.

Rivas, E.K de, 1972, "On the use of non-uniform grids in finitedifference equations", J. Comput. Phys., Vol. 10, pp. 202-210.

Sadri, R.M. \& Floryan, J.M., 2002a, "Accurate Evaluation of the loss coefficient and the entrance length of the inlet region of a channel", Journal of Fluids Engineering, Vol.124, pp. 685-693.

Sadri, R.M. \& Floryan, J.M., 2002b, "Entry flow in a chanel" Computers \& Fluids, Vol. 31, pp. 133-137.

Santos, R.G., 2004, Numerical Study of the flow at a sharp-edged entrace to a pipe in laminar regime (in Portuguese) Master Thesis, FEM, UNICAMP.

Schlichting, H., 1979, Boundary-Layer Theory, McGraw-Hill Book Company, New York.

Shah, R.K. \& London, A.L., 1978, Laminar Flow Forced Convection in Ducts, Academic Press, New York.

Sisavath, S., Jing, X., Pain, C.C. and Zinnerman, R.W., 2002, "Creeping Flow Through an Axisymmetric Sudden Contracrion or Expansion", Journal of Fluids Engineering, Vol. 124, pp. 273-278.

Sparrow, E.W. \& Anderson, C.E., 1977, "Effect of Upstream Flow Processes on Hydrodynamic Development in a Duct", Journal of Fluids Engineering, Vol. 99, pp. 556-560.

Sylvester, N.D. \& Rosen, S.L., 1970, "Laminar Flow in the Entrace Region of a Circular Tube", AIChE Journal, Vol. 16, No. 6, pp. 964-971.

Uribe, E.B.O., 2002, Evaluation of Discretization Schemes in the Solution of Navier-Stokes Equations and Application to the Problem of the Flow in the Entrance Region of Plane Channels (in Portuguese), Doctoral Thesis, FEM, UNICAMP.

Vennard, J.K. \& Street, R.L., 1978, Elementary fluid mechanics (in Portuguese), Guanabara Dois, Rio de Janeiro, 5 th ed.

Vilela, C.A.deA., 2001, Simulation of flows in generalized coordinates using the finite volume method with the UNIFAES discretization scheme (in Portuguese), Doctoral Thesis, FEM, UNICAMP.

Vrentas, J.S. \& Duda, J.L., 1973, "Flow of a Newtonian Fluid Through a Sudden Contraction", Appl. Sci. Res., Vol. 28, pp. 241-260. 